\title{
Comparative Analysis Study of ATC-40 and SNI 1726-2012 Guidelines For Beam Structure Performance and Column Trans Studio Apartments Applications Using Dynamic Response Spectrum Analysis Methods
}

\author{
Agyanata Tua Munthe, Abdul Gafur \\ Faculty of Engineering, Mercu Buana University Jakarta, Indonesia \\ Agyanata.umb@gmail.com, abdulgafur.1995@gmail.com
}

\begin{abstract}
The earthquake that often hit Indonesia caused thousands of lives and caused damage to buildings. These earthquakes often occur because Indonesia is in two regions, namely the Pacific earthquake path (Circum Pacific Earthquake Belt) and the Asian earthquake lane (Trans Asiatic Earthquake Belt). Earthquake disasters cause damage to building structures. When an earthquake occurs, it is expected that the building can accept earthquake force at a certain level without significant damage to its structure. In general, earthquake analysis is divided into two major parts, namely static earthquake analysis and dynamic earthquake analysis. In buildings that are very high, irregular, multilevel, and buildings that require enormous accuracy are used dynamic analysis planning, which consists of a variety of spectral response analysis and dynamic time response dynamic analysis. This study aims to determine the building's security in terms of displacement, drift, and base shear. The method used is a dynamic analysis of the response spectrum using the ETABS program. The maximum total drift in the $\mathrm{X}$ direction is $0.0200475 \mathrm{~m}$ and in the $\mathrm{Y}$ direction is $0.020405 \mathrm{~m}$, so the building is safe against ultimate boundary performance $(0.02 \mathrm{~h})$ and service boundary performance $\{(0.03 / \mathrm{R}) \mathrm{x} \mathrm{h}\}$. So that the displacement in the building does not exceed the maximum displacement, the building is safe from earthquake plans.

Keyword: Response Spectrum, ATC-40, SNI 1726-2012, Comparative Analysis
\end{abstract}

\section{Introduction}

Natural disasters are natural events that can occur at any time and anywhere and in unexpected periods. Natural disasters also cause negative impacts such as material and non-material losses for people's lives.

Earthquake planning on the Trans Studio Cibubur Apartment building uses SNI 1726-2012 regulations. Evaluation of the structure is needed to determine the security of the building structure. This regulation is used to determine the design parameters for earthquake risk, earthquake risk.

In this analysis research method uses the earthquake response spectrum plan as a basis for determining the response. In the spectrum response analysis is only used to determine the shear force of the nominal dynamic level due to the effect of the earthquake plan. Internal forces in building structure elements are obtained from 3-dimensional analysis based on equivalent static earthquake loads.

\section{Methodology}

\section{Stages of Analysis}

Make a spectrum response curve based on various lateral force distribution patterns, especially those equivalent to the distribution of the inertia force, so that it is expected that the deformation that occurs is almost the same or close to the deformation that occurs due to an earthquake. Because the nature of the earthquake is uncertain, several lateral loading patterns need to be made to get the most decisive conditions.

The estimated magnitude of lateral displacement during planned earthquake (displacement target). The control point is pushed up to the level of displacement, which reflects the maximum displacement caused by the intensity of the specified earthquake plan.

Evaluating the level of structure performance when the control point is precisely at the target displacement: is the main thing of performance-based planning. The structural components and their behavioral actions can be considered satisfactory if they meet the criteria that were set from the start, both for deformation and strength requirements. Because the components evaluated are relatively large in number, therefore this process must be done entirely by the computer. 

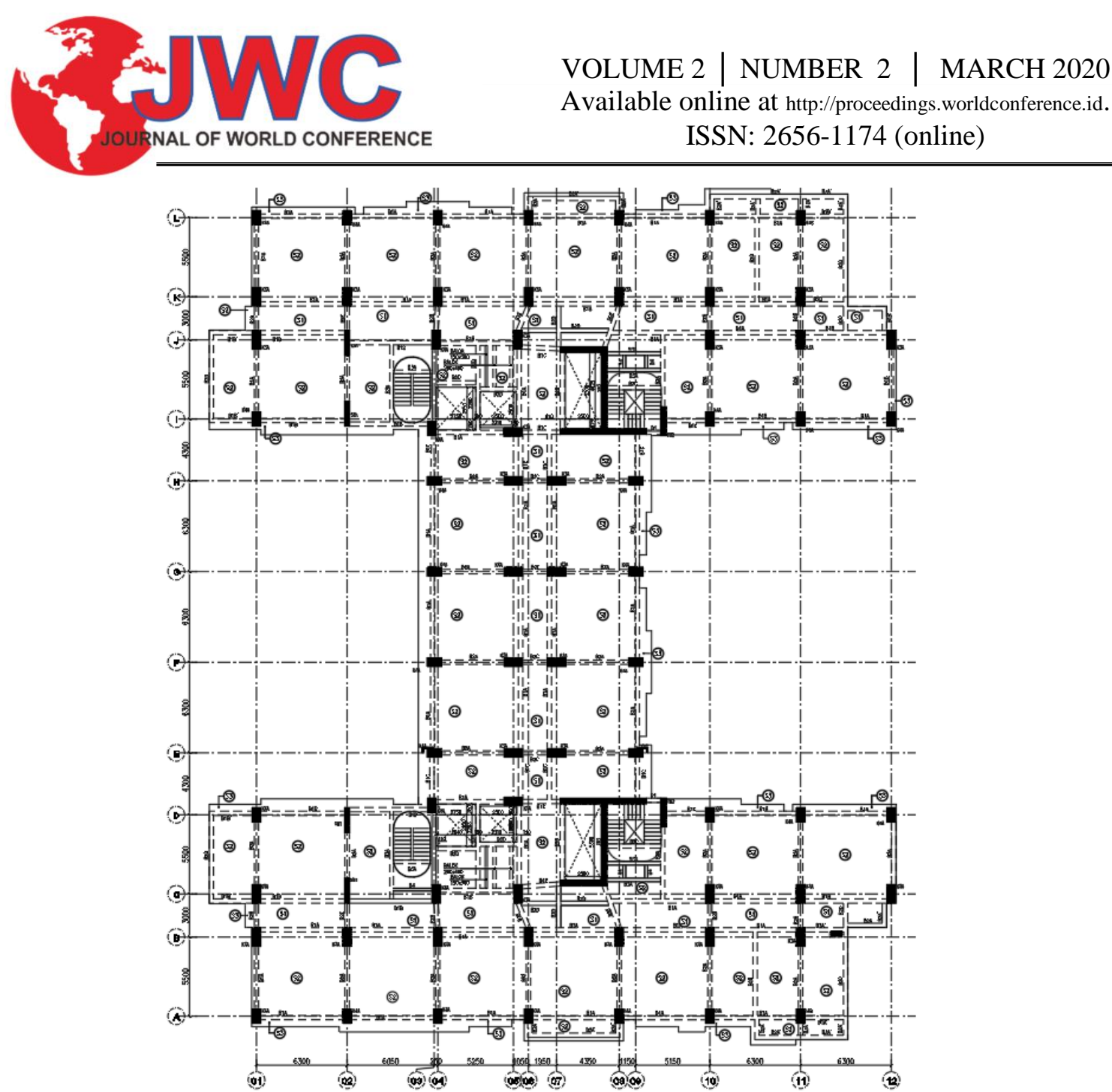

Figure 1. Structure Plan of Tower C at Transtudio Cibubur Project Source: PT WIKA Project Document

\section{Structure Geometry}

a. Building Name

b. Location

c. Building Function: Apartment

d. Site Type: Soft Soil

e. Number of Floors: 30 Stories

f. Floor to floor height

Floor $1 \sim$ Floor 2

Floor $2 \sim$ Floor 29

: $3,5 \mathrm{~m}$

$: 3.2 \mathrm{~m}$

: $3.2 \mathrm{~m}$

g. Total Height: $96 \mathrm{~m}$

h. Seismic Restraint System: Special moment reinforced concrete frame bearers

\section{Earthquake Loads}

Based on SNI 1726-2012, the earthquake loading for this building located in Harjamukti, Depok has the following data. 


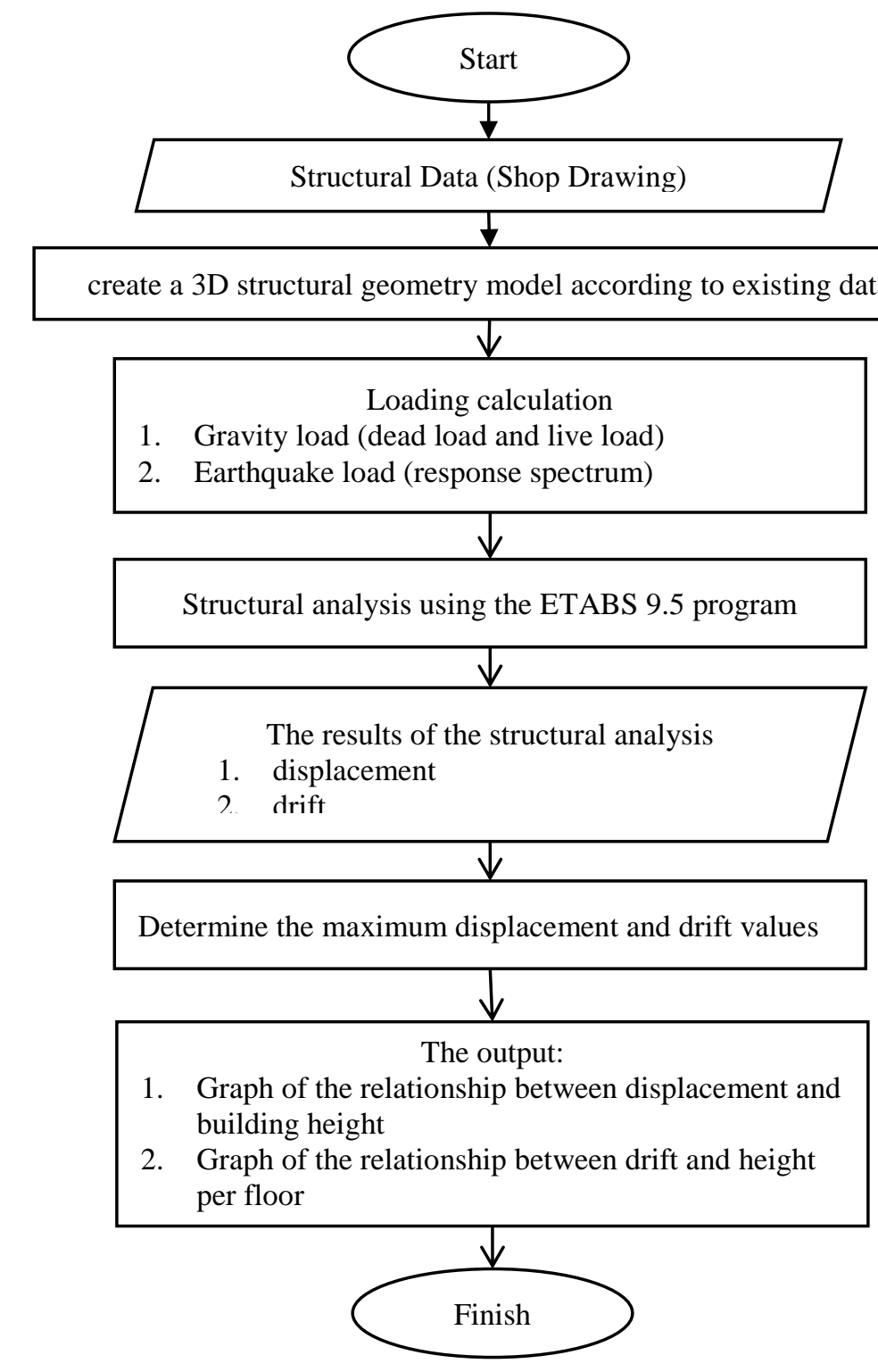

Figure 1. Research Flow Diagram

Source: Author's Process, 2019

\section{Risk Categories and Priority Factors}

The function of the building as a Residential Apartment. Based on table 1 and table 2 of SNI 1726-2012, the risk category value $=\mathrm{II}$ and the priority factor $(\mathrm{Ie})$ are obtained as 1.0

\section{Site Class}

Due to limited data, this report uses the type of soil used as stated in the as-built drawing, namely soft soil. In table 3 SNI 1726-2012 soft soil is categorized as SE.

\section{Earthquake Parameter Value}

The earthquake parameter values are taken from the Indonesian spectra Design online application which can be accessed through http://puskim.go.id/Aplikasi/desainspektraindonesia2011 on January 3, 2020, by inputting the name of the city or the coordinates of the location of the building site type of soft rock. 


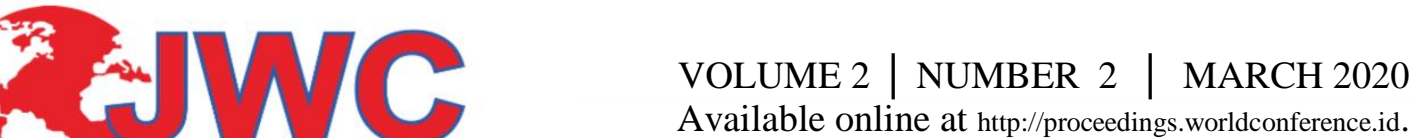

Available online at http://proceedings.worldconference.id. ISSN: 2656-1174 (online)

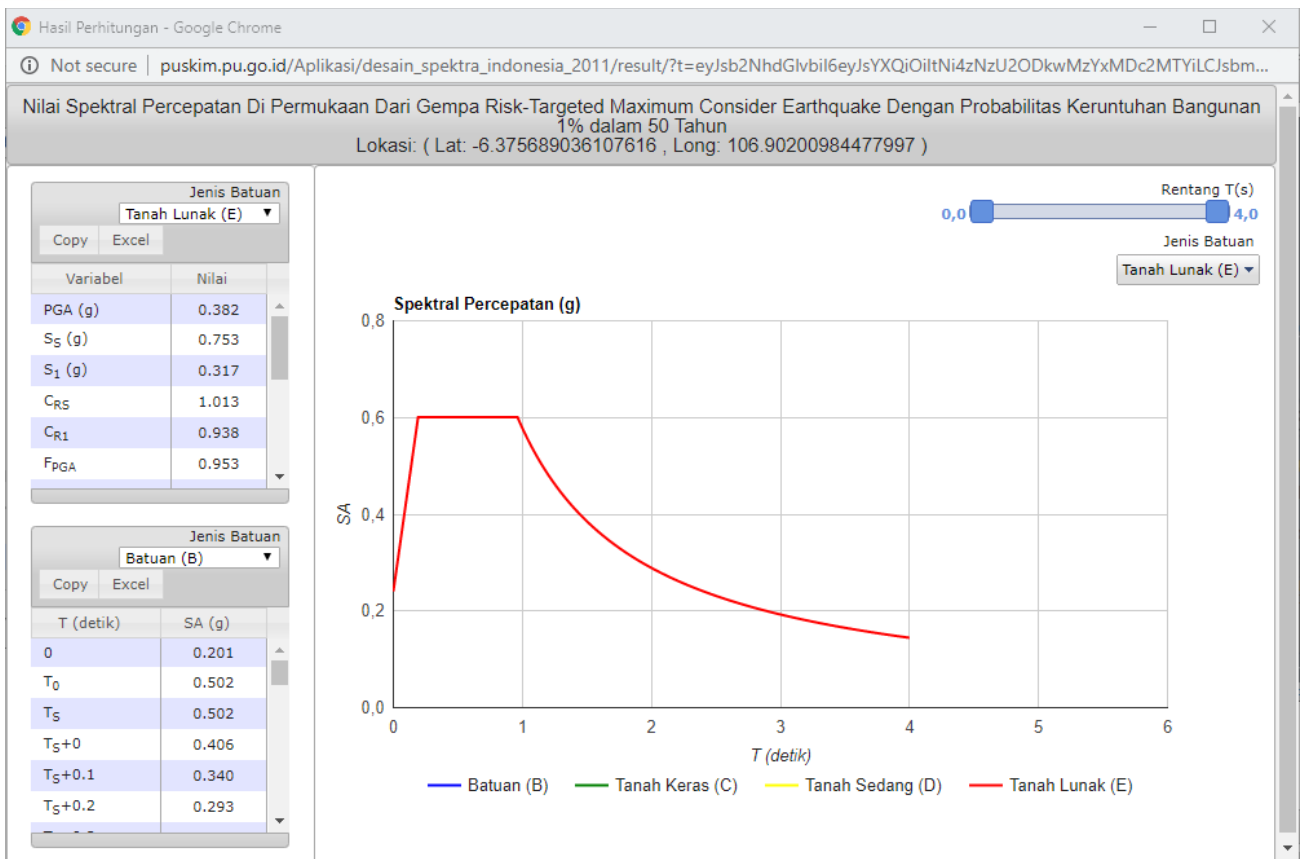

Figure 3. Spectral Acceleration for Harjamukti Area, Depok Source: http://puskim.go.id/Aplikasi/desainspektraindonesia2011

Table 1. Spectral Value of Earthquake Acceleration on the Surface of Harjamukti Area, Depok

\begin{tabular}{|l|l|}
\hline Variable & Nilai \\
\hline PGA (g) & 0.382 \\
\hline SS $($ g) & $\mathbf{0 . 7 5 2}$ \\
\hline S1 (g) & $\mathbf{0 . 3 1 7}$ \\
\hline CRS & 1.012 \\
\hline CR1 & 0.938 \\
\hline FPGA & 1 \\
\hline FA & 1 \\
\hline FV & 1 \\
\hline PSA (g) & 0.382 \\
\hline SMS (g) & 0.752 \\
\hline SM1 (g) & 0.317 \\
\hline SDS (g) & 0.501 \\
\hline SD1 (g) & 0.211 \\
\hline T0 (second) & 0.084 \\
\hline TS (second) & 0.422 \\
\hline
\end{tabular}

Source: Indonesian Spectra Design Application 
VOLUME 2 | NUMBER 2 | MARCH 2020

Available online at http://proceedings.worldconference.id.

ISSN: 2656-1174 (online)

Tabel 2. The Spectral Period Value of Earthquake Acceleration on Harjamukti Surface, Depok

$$
\text { T }
$$

\begin{tabular}{l|l}
$\mathrm{T}$ (second) & $\mathrm{SA}(\mathrm{g})$
\end{tabular}

0

\begin{tabular}{|l|l|}
\hline T & 0.24 \\
\hline T0 & 0.6 \\
\hline TS & 0.6 \\
\hline
\end{tabular}

\begin{tabular}{|l|l}
\hline TS+0 & 0.544 \\
\hline TS & 0.1
\end{tabular}

\begin{tabular}{l|l} 
TS +0.1 & 0.497 \\
\hline TS & 0.2 \\
\hline
\end{tabular}

\begin{tabular}{l|l} 
TS +0.2 & 0.458 \\
\hline TS & 0.3
\end{tabular}

\begin{tabular}{l|l} 
TS +0.3 & 0.424 \\
\hline TS +0.4 & 0.395
\end{tabular}

\begin{tabular}{l|l}
\hline $\mathrm{TS}+0.4$ & 0.395 \\
\hline
\end{tabular}

\begin{tabular}{l|l}
\hline TS +0.5 & 0.37 \\
\hline
\end{tabular}

\begin{tabular}{l|l}
\hline TS +0.6 & 0.347 \\
\hline TS
\end{tabular}

\begin{tabular}{l|l}
\hline $\mathrm{TS}+0.7$ & 0.328 \\
\hline $\mathrm{TS}+0.8$ & 0.31
\end{tabular}

\begin{tabular}{|l|l|}
\hline TS +0.8 & 0.31 \\
\hline TS +0.9 & 0.294 \\
\hline TS +1
\end{tabular}

\begin{tabular}{l|l}
$\mathrm{TS}+1$ & 0.28 \\
\hline
\end{tabular}

\begin{tabular}{l|l}
\hline TS+1.1 & 0.267 \\
\hline TS+1.2
\end{tabular}

\begin{tabular}{|l|l|}
\hline TS+1.2 & 0.255 \\
\hline & 0.244 \\
\hline
\end{tabular}

\begin{tabular}{l|l}
$\mathrm{TS}+1.3$ & 0.244 \\
\hline
\end{tabular}

\begin{tabular}{l|l}
$\mathrm{TS}+1.4$ & 0.235 \\
\hline $\mathrm{TS}+1.5$ & 0.225
\end{tabular}

\begin{tabular}{l|l} 
TS+1.5 & 0.225 \\
\hline
\end{tabular}

\begin{tabular}{|l|l|}
\hline TS+1.6 & 0.217 \\
\hline TS+1.7 & 0.209
\end{tabular}

\begin{tabular}{l|l} 
TS+1.7 & 0.209 \\
\hline TS+1.8
\end{tabular}

\begin{tabular}{|l|l|}
\hline TS +1.8 & 0.202 \\
\hline TS +1.9 & 0.195
\end{tabular}

\begin{tabular}{|l|l|}
\hline TS+1.9 & 0.195 \\
\hline TS+2 & 0.189 \\
\hline TS+2.1 & 0.183 \\
\hline TS+2.2 & 0.177 \\
\hline TS+2.3 & 0.172 \\
\hline TS +2.4 & 0.167 \\
\hline TS+2.5 & 0.162 \\
\hline TS +2.6 & 0.158 \\
\hline TS+2.7 & 0.153 \\
\hline TS +2.8 & 0.15 \\
\hline TS +2.9 & 0.146 \\
\hline 4 & 0.144 \\
\hline
\end{tabular}

Source : Indonesian Spectra Design Application

\section{Seismic Design Category}

The building seismic design category is taken from the value of the acceleration response parameter in the short period (SDS) and the acceleration response parameter in the 1 second period (SD1). Based on table 4.3, the SDS value $=0.501 \mathrm{~g}$ and SD1 = $0.211 \mathrm{~g}$ are obtained. Then the seismic design category is determined based on the following table: 


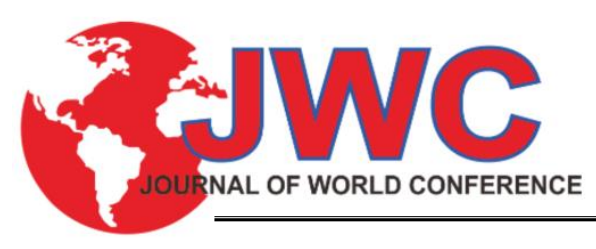

VOLUME 2 | NUMBER 2 | MARCH 2020

Available online at http://proceedings.worldconference.id.

ISSN: 2656-1174 (online)

Table 3. Building Seismic Design Risk Categories

\begin{tabular}{|l|l|l|l|}
\hline \multicolumn{2}{|l|}{ Value } & \multicolumn{2}{c|}{ Risk Category } \\
\cline { 3 - 4 } \multicolumn{2}{l|}{} & I or II or III & IV \\
\hline SDS $=501 \mathrm{~g}$ & SDS $>/ 0.500$ & D & D \\
\hline SD1 $=0.211 \mathrm{~g}$. & SD1 $>/ 0.200 \mathrm{~g}$ & D & D \\
\hline \multicolumn{2}{|c|}{ Source $:$ SNI 1726-2012 }
\end{tabular}

\section{Structure System}

The existing structural system in principle uses a frame system to hold gravity loads, while lateral loads caused by earthquakes are shouldered using a bending mechanism. The moment bearing frame system is divided into three namely SPRMB (Ordinary Moment Resisting Frame System) SPRMM (Order System

Medium Moment Bearer, SPRMK (Special Moment Bearer Frame System).

The structure reviewed in this study has a $\mathrm{D}$ type seismic design category

then based on table 4.6 the coefficient of response modification obtained (R), the strength of the system over $(\Omega 0)$, the deflection magnification factor $(\mathrm{Cd})$. The structural parameter data for the earthquake barrier system is as follows:

Table 4. Faktor R, $\Omega 0$, dan Cd

\begin{tabular}{|c|c|c|c|c|c|c|c|c|}
\hline \multirow[t]{3}{*}{ Earthquake Resistance System } & \multirow{3}{*}{$\begin{array}{l}\begin{array}{l}\text { Response } \\
\text { modification } \\
\text { coefficient }\end{array} \\
\mathrm{R}\end{array}$} & \multirow{3}{*}{$\begin{array}{l}\text { More } \\
\text { Powerful } \\
\text { Factor } \\
\Omega 0\end{array}$} & \multirow{3}{*}{$\begin{array}{l}\text { Deflection } \\
\text { Magnification } \\
\text { Factor } \\
\mathrm{Cd}\end{array}$} & \multirow{2}{*}{\multicolumn{5}{|c|}{$\begin{array}{l}\text { Limitations of Structural Systems and } \\
\text { Height Limits of Structures } \\
\text { Kategori Desain Seismik }\end{array}$}} \\
\hline & & & & & & & & \\
\hline & & & & $\mathrm{B}$ & $\overline{\mathrm{C}}$ & $\mathrm{D}$ & $\bar{E}$ & $\mathrm{~F}$ \\
\hline \multicolumn{9}{|l|}{ Moment bearer frame system } \\
\hline $\begin{array}{l}\text { 6. Moment-bearing reinforced } \\
\text { concrete frame }\end{array}$ & 5 & 3 & $41 / 2$ & TB & TI & TI & TI & TI \\
\hline $\begin{array}{l}\text { 7. The ordinary moment-bearing } \\
\text { reinforced concrete frame }\end{array}$ & 3 & 3 & $21 / 2$ & TB & TI & TI & TI & TI \\
\hline $\begin{array}{l}\text { 8. Special moment-bearing } \\
\text { reinforced concrete frame }\end{array}$ & 8 & 3 & $51 / 2$ & TB & TB & TB & TB & TB \\
\hline
\end{tabular}

The structural system used in this building is a medium moment reinforced concrete frame system. Based on the discussion in Chapter II of Table 2.10 , the parameter values of $\mathrm{R}, \Omega 0$, Cd are respectively $8,3,51 \frac{1}{2}$.

\section{Make a Combination Load}

In the ultimate design, the load combination given to the building structure must refer to SNI 1727: 2013 so that the load combination given to the building is a combination of 1 to a combination of 18 . To facilitate the calculation of reinforcement, 1 (one) Gravity load is added with a value of $1.2 \mathrm{DL}+1 \mathrm{LL} .18$ (eighteen) combinations and 1 gravity load combination as follows:
1. Comb 1
2. Comb 2
3. Comb 3
4. Comb 4
5. Comb 5
6. Comb 6
7. Comb 7
8. Comb 8
9. Comb 9
10. Comb 10
11. Comb 11
12. Comb 12
13. Comb 13
14. Comb 14
15. Comb 15
$: 1.4 \mathrm{DL}$
: 1.2 DL+1.6 LL
$: 1.2 \mathrm{DL}+1.0 \mathrm{EQx}+0.3 \mathrm{EQy}+0.5 \mathrm{LL}$
$: 1.2 \mathrm{DL}+1.0 \mathrm{EQx}-0.3 \mathrm{EQy}+0.5 \mathrm{LL}$
: $1.2 \mathrm{DL}-1.0 \mathrm{EQx}+0.3 \mathrm{EQy}+0.5 \mathrm{LL}$
$: 1.2 \mathrm{DL}-1.0 \mathrm{EQx}-0.3 \mathrm{EQy}+0.5 \mathrm{LL}$
$: 1.2 \mathrm{DL}+1.0 \mathrm{EQy}+0.3 \mathrm{EQx}+0.5 \mathrm{LL}$
$: 1.2 \mathrm{DL}+1.0 \mathrm{EQy}-0.3 \mathrm{EQx}+0.5 \mathrm{LL}$
$: 1.2 \mathrm{DL}-1.0 \mathrm{EQy}+0.3 \mathrm{EQx}+0.5 \mathrm{LL}$
$: 1.2 \mathrm{DL}-1.0 \mathrm{EQy}-0.3 \mathrm{EQx}+0.5 \mathrm{LL}$
: $0.9 \mathrm{DL}+1.0 \mathrm{EQx}+0.3 \mathrm{EQy}$
: $0.9 \mathrm{DL}+1.0 \mathrm{EQx}-0.3 \mathrm{EQy}$
: $0.9 \mathrm{DL}-1.0 \mathrm{EQx}+0.3 \mathrm{EQy}$
: $0.9 \mathrm{DL}-1.0 \mathrm{EQx}-0.3 \mathrm{EQy}$
: $0.9 \mathrm{DL}+1.0 \mathrm{EQy}+0.3 \mathrm{EQx}$
16. Comb 16
$: 0.9 \mathrm{DL}+1.0 \mathrm{EQy}-0.3 \mathrm{EQx}$ 


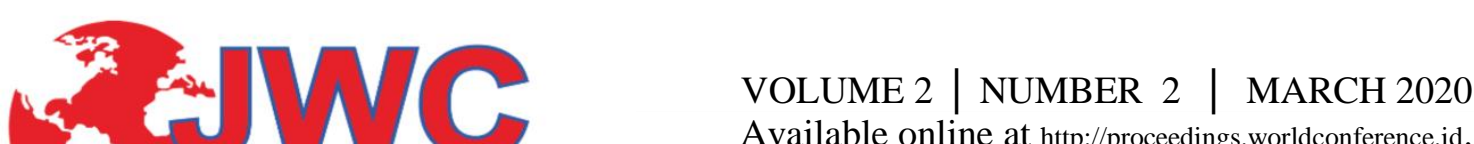

Available online at http://proceedings.worldconference.id.

ISSN: 2656-1174 (online)

17. Comb 17

18. Comb 18

19. Graff

$: 0.9 \mathrm{DL}-1.0 \mathrm{EQy}+0.3 \mathrm{EQx}$

: $0.9 \mathrm{DL}-1.0 \mathrm{EQy}-0.3 \mathrm{EQx}$

: 1.2 DL +1.0 LL

\section{Fundamental Natural Vibration Time (T)}

Following SNI 1726-2012 earthquake regulations article 7.8.2 for determining the period of natural vibration structure (T) using the following coefficients:

a. Period Upper Limit Coefficient

Table 5. The coefficient for the upper limit for the period calculated

\begin{tabular}{|l|l|}
\hline Parameters design spectral response acceleration at 1 second, & Coefficient, $C_{u}$ \\
$S_{D 1}$ & \\
\hline $\mathbf{0 . 2}$ & $\mathbf{1 , 5}$ \\
\hline
\end{tabular}

Source: SNI 1726-2012 Pasal 2.2.11c

Period upper limit coefficient $\boldsymbol{C}_{\boldsymbol{u}}$ with $S_{D l} 0,211 \mathrm{~g}$ is $\mathbf{1 . 5}$

b. Parameter Value for Approach Period $C_{t}$ dan $x$

Table 6. Parameter Value for Approach Period $\mathrm{C}_{\mathrm{t}}$ and $\mathrm{x}$

\begin{tabular}{|l|l|l|}
\hline Structure Type & $C_{t}$ & $x$ \\
\hline Moment-bearing concrete frame & 0,0466 & 0,9 \\
\hline
\end{tabular}

Source: SNI $1726: 2012$ Pasal 2.2.11c

Approximate parameter $\boldsymbol{C}_{\boldsymbol{t}}$ adalah $\mathbf{0 . 0 4 6 6}{ }^{\mathrm{a}}$

Approximate parameter $\mathbf{x}$ adalah $\mathbf{0 . 9}$

a. The period of fundamental approach $\left(T_{a}\right)$ in second can be determined by the following equation:

$$
\begin{gathered}
T_{a}=C_{t} h_{n}{ }^{x} \\
T_{\text {maks }}=C_{u} \cdot T_{a} \text { atau } T_{a}<T_{\max }=C_{t} \times h_{n}{ }^{x} \times C_{u}
\end{gathered}
$$

where:

$C_{t}$ and $x=$ coefficient based on the type of structure used

$C_{u} \quad=$ the coefficient for the upper bound for the calculated period

$h_{n} \quad=$ the height of the structure in meters, above the base of the highest level of the structure.

b. Fundamental period value limits obtained as follows :

$\boldsymbol{T}_{a}\left(\boldsymbol{T}_{\min }\right) \quad=0.0466 \times 96.1^{0.9}=\mathbf{2 . 8 3 4 1 9 7}$ detik

$\boldsymbol{T}_{\text {maks }} \quad=1.5 \times 2.834197=\mathbf{4 . 2 5 1 2 9 5 5}$ detik

Controls the fundamental vibration time limitation viz:

Jika $T x$ dan $T y<T_{\text {maks }}$, then the vibration time limitation control is fulfilled.

Jika $T x$ dan $T y>T_{m a k s}$, then the vibration time limitation control is Not fulfilled.

c. The natural period values from the ETABS program results are as follows :

Table 7. Natural Period Results of the Etabs Program

\begin{tabular}{|l|l|l|l|l|}
\hline & $T_{x}$ & $\mathbf{T}_{\mathbf{y}}$ & Ket \\
\hline Design & & $\mathbf{3 . 9 3 3}$ second & $\mathbf{3 . 9 3 3}$ second & Fulfilled \\
\hline
\end{tabular}

\section{Results and Discussion}

Based on SNI 1726: 2012 earthquake 7.8.6 in the determination of intersections between floors the design level $(\Delta)$ must be calculated as the difference in deflection at the center of mass at the top and bottom levels reviewed. Deflection of the center of mass at $\mathrm{x}(\delta \mathrm{x})(\mathrm{mm})$ must be determined according to the following equation: 


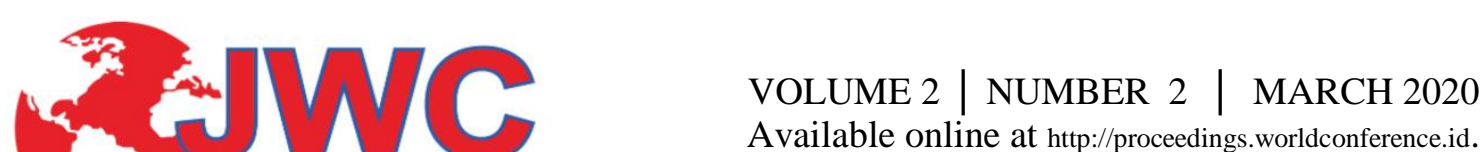

Available online at http://proceedings.worldconference.id.

ISSN: 2656-1174 (online)

Dengan :

$$
\delta_{x}=\frac{C_{d} \delta_{x e}}{I_{e}}
$$

$C_{d}=$ The magnification factor of the deflection amplification is determined by the type of seismic force structure selected

(SRPMK = 8).

$\Delta x e=$ deflection at the required location $(\mathrm{mm})$.

Ie = earthquake priority factor, (Kategori resiko II =1)

The type of structure is a structure using shearwall (shear wall), then the deviation between floors allows for risk category II then using the formula:

$\Delta_{\mathrm{a}}=0.015 \times h_{s x} / \rho$

Following are the displacement data from ETABS 2017:

Table 8. Deviation Between Floor

\begin{tabular}{|c|c|c|c|c|c|c|}
\hline Floor & $\mathbf{H}(\mathbf{m})$ & $\begin{array}{c}\text { Cumulative } \\
\text { H (m) }\end{array}$ & $\begin{array}{c}\text { UX } \\
\text { Response } \\
\text { Spectrum }\end{array}$ & $\begin{array}{c}\text { UY } \\
\text { Response } \\
\text { Spectrum }\end{array}$ & $\begin{array}{c}\text { Terms } \\
{[(0,015 \text { hsx }) / \rho]}\end{array}$ & Information \\
\hline Floor30 & 3.2 & 96.3 & 0.0200475 & 0.020405 & 0.71615 & secure \\
\hline Floor29 & 3.2 & 93.1 & 0.0208945 & 0.0211255 & 0.69154 & secure \\
\hline Floor28 & 3.2 & 89.9 & 0.0217415 & 0.021703 & 0.66692 & secure \\
\hline Floor27 & 3.2 & 86.7 & 0.02266 & 0.0222695 & 0.64231 & secure \\
\hline Floor26 & 3.2 & 83.5 & 0.0235235 & 0.0227645 & 0.61769 & secure \\
\hline Floor25 & 3.2 & 80.3 & 0.024288 & 0.0232045 & 0.59308 & secure \\
\hline Floor24 & 3.2 & 77.1 & 0.0247445 & 0.023386 & 0.56846 & secure \\
\hline Floor23 & 3.2 & 73.9 & 0.0252395 & 0.023639 & 0.54385 & secure \\
\hline Floor22 & 3.2 & 70.7 & 0.0256245 & 0.023804 & 0.51923 & secure \\
\hline Floor21 & 3.2 & 67.5 & 0.0259435 & 0.0239085 & 0.49462 & secure \\
\hline Floor20 & 3.2 & 64.3 & 0.0261965 & 0.0239635 & 0.47000 & secure \\
\hline Floor19 & 3.2 & 61.1 & 0.0264055 & 0.02398 & 0.44538 & secure \\
\hline Floor18 & 3.2 & 57.9 & 0.0265705 & 0.0239525 & 0.42077 & secure \\
\hline Floor17 & 3.2 & 54.7 & 0.0267025 & 0.0238865 & 0.39615 & secure \\
\hline Floor16 & 3.2 & 51.5 & 0.026829 & 0.0238095 & 0.37154 & secure \\
\hline Floor15 & 3.2 & 48.3 & 0.0268015 & 0.0236005 & 0.34692 & secure \\
\hline Floor14 & 3.2 & 45.1 & 0.026884 & 0.0234685 & 0.32231 & secure \\
\hline Floor13 & 3.2 & 41.9 & 0.026928 & 0.023287 & 0.29769 & secure \\
\hline Floor12 & 3.2 & 38.7 & 0.0269445 & 0.0230505 & 0.27308 & secure \\
\hline Floor11 & 3.2 & 35.5 & 0.0269115 & 0.022748 & 0.24846 & secure \\
\hline Floor10 & 3.2 & 32.3 & 0.026807 & 0.0223355 & 0.22385 & secure \\
\hline Floor9 & 3.2 & 29.1 & 0.0265705 & 0.0217855 & 0.19923 & secure \\
\hline Floor8 & 3.2 & 25.9 & 0.0261525 & 0.0210485 & 0.17462 & secure \\
\hline Floor7 & 3.2 & 22.7 & 0.025454 & 0.0200585 & 0.15000 & secure \\
\hline Floor6 & 3.2 & 19.5 & 0.024332 & 0.018744 & 0.12538 & secure \\
\hline Floor5 & 3.2 & 16.3 & 0.0227755 & 0.017193 & 0.10077 & secure \\
\hline Floor4 & 3.2 & 13.1 & 0.020504 & 0.01518 & 0.07615 & secure \\
\hline Floor3 & 3.2 & 9.9 & 0.0172975 & 0.0126555 & 0.05154 & secure \\
\hline Floor2 & 3.2 & 6.7 & 0.0128865 & 0.0095095 & 0.02692 & secure \\
\hline Floor1 & 3.5 & 3.5 & 0.0056045 & 0.004411 & 0 & secure \\
\hline
\end{tabular}




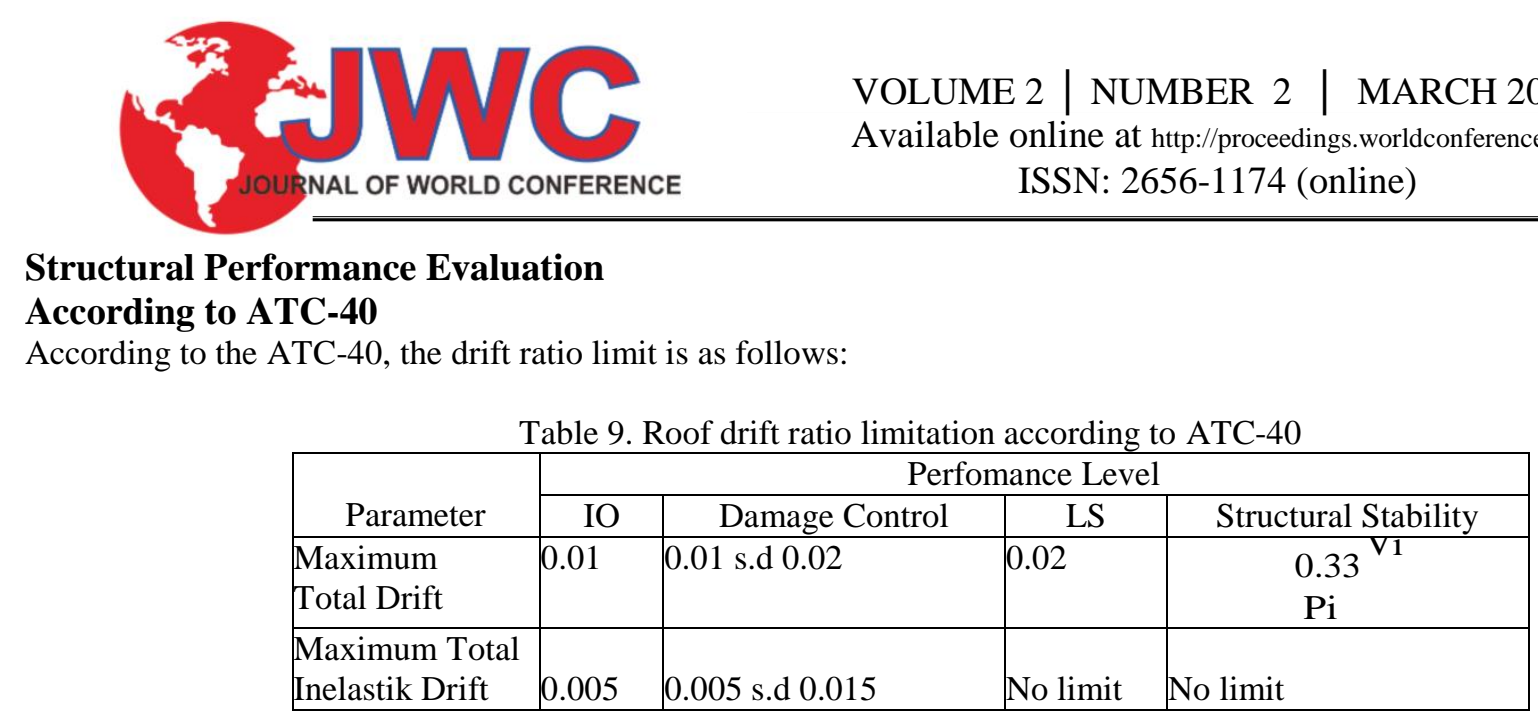

\section{Building performance according to ATC-40 for $\mathrm{X}$ direction}

Maximum Drift $=$ Dt $/$ Htotal $=0.0200475 / 96.3=2.0817$

So the level of building performance is Life Safety

Maximum Inelastic Drift $=$ Dt-D1 / Htotal $=(0.0200475-0.0056045) / 96.3=1.4997$

The level of performance of nonlinear buildings is Life Safety

\section{Building performance according to ATC-40 for $\mathrm{Y}$ direction}

Maximum Drift $=$ Dt $/$ Htotal $=0.020405 / 96.3=2.1188$

So the level of building performance is Life Safety

Maximum Inelastic Drift $=$ Dt-D1 $/$ Htotal $=0.020405-0.004411) / 96.3=1.6608$

The level of performance of nonlinear buildings is Life Safety

Note: $\mathrm{Dt}=$ roof displacement

\section{Conclusions}

$$
\text { D1 = 1st floor displacement }
$$

After analyzing using the spectrum response method in chapter 4, the writer can draw the following conclusions:

1. Drift between $X$ and $Y$ directions is declared safe against service boundary performance.

2. Drift between $X$ and $Y$ directions is declared safe against Ultimate boundary performance.

3. The results of the maximum displacement X-direction obtained $0.0200475 \mathrm{~m}$ and $\mathrm{Y}$ direction obtained $0.020405 \mathrm{~m}$ so that it still meets the maximum limit $[(0.015 \mathrm{hsx}) / \rho]=0.71615 \mathrm{~m}$.

4. According to the ATC-40 performance level based on Dynamic Analysis of Response Spectrums in the X and Y direction the building performance level is Life Safety.

\section{Reference}

[1] Andriyanto Firman, (2014). Evaluasi Kinerja Struktur Gedung Bertingkat Dengan Analisis Riwayat Waktu Terhadap Drift dan Displacement Menggunakan Software Etabs, Universitas Sebelas Maret

[2] Applied Technology Council 40 (1996). Seismic Evaluation and Retrofit of Concrete Buildings. Volume 1. Redwood City, California, U.S.A.

[3] ASCE (2000). FEMA 356 - Prestandard And Commentary For The Seismic Rehabilitation Of Buildings. Federal Emergency Management Agency. Washington, D.C.

[4] Baiquny Febbrian, Donny (2014), Evaluasi Kinerja Gaya Gempa Pada Gedung Bertingkat Dengan Analisis Respon Spektrum Berdasarkan Base Shear, Displacement, dan Drift, Universitas Sebelas Maret

[5] Luthfi Rifa'i, Muhammad (2014), Evaluasi Kinerja Struktur Pada Gedung Bertingkat Dengan Analisis Dinamik Respon Spektrum Menggunakan Software Etabs, Universitas Sebelas Maret

[6] Marwato Ary, (2014), Evaluasi Kinerja Struktur Gedung 10 Floor Dengan Analisis Pushover Terhadap Drift Dan Displacement Menggunakan Software Etabs, Universitas Sebelas Maret

[7] N. Patil, Mahes (2015), Seismic Analysis of Multistoried Building, M. Tech. (Structure) SVNIT

[8] Nurhadi Muhammad, (2014), Evaluasi Kinerja Gaya Gempa Pada Struktur Gedung Bertingkat dengan Analisis Pushover Berdasar Pada Drift dan Displacement Menggunakan Etabs, Universitas Sebelas Maret 


\section{Biographies}

Abdul Gafur is a Staff Engineer in the National Construction Company in PT Wijaya Karya Bangunan Gedung in Cibubur. He is was born in April 1, 1995. After his high school education at SMK 26 Pembangunan in Jakarta, he has continued his civil engineering education at Mercu Buana Unversity at Kranggan, Depok.

Agyanata Tua Munthe was born on March 21, 1981. After his highschool education at BPK Penabur Senior High School in Bandar Lampung, he is continued his civil engineering education at Atmajaya University, Special Region of Gadjah Mada, Special Region of Yogyakarta. Currently active as a lecturer at Mercu Buana University, Jakarta as a lecturer with a concentration in structure and currently active as a Project Manager to handling several construction projects. 